\title{
The particular case of sleep-disordered breathing in syndromic patients
}

\author{
B. Fauroux'1, A. Amaddeo ${ }^{2}$ \\ 1 Pediatric pulmonologist, Head of the Pediatric Non-invasive Ventilation and Sleep Unit, \\ Necker Hospital, Paris, France \\ 2 Pediatric pulmonologist, Pediatric Non-invasive Ventilation and Sleep Unit, Necker Hospital, \\ Paris, France
}

\section{ABSTRACT}

Classical childhood obstructive sleep apnea syndrome (OSAS) is relatively common, affecting approximately $1 \%$ to $2 \%$ of children between the ages of 3 to 6 years. Adenotonsillar hypertrophy is the prime cause, and adenotonsillectomy is the first-line treatment, leading to cure in most cases.

However, OSAS is also found in some more rare but very numerous disorders, including craniofacial or upper airway deformities such as Pierre Robin sequence, Franceschetti syndrome, craniofacial stenosis, achondroplasia, Down syndrome, Prader-Willi syndrome, and mucopolysaccharidosis. Unlike in classical childhood OSAS, anatomical and functional upper airway abnormalities are the main pathophysiological determinants, clinical symptoms of sleep-disordered breathing are often slight or absent, and the syndrome is usually more severe than classical childhood OSAS and can be observed at any age; systematic sleep study is therefore justified. Isolated adenotonsillectomy is rarely able to cure the OSAS.

Management should be multidisciplinary, including, severally or together, a pediatric ENT surgeon, pediatric maxillofacial surgeon, orthodontist, pediatric neurosurgeon, pediatric sleep specialist and an expert in pediatric non-invasive ventilation because of the frequent need for nocturnal respiratory support by non-invasive continuous positive airway pressure.

It is important to be aware of these rare pathologies that may underlie OSAS, to enable early screening for sleep-disordered breathing and adapted therapy.

\section{KEYWORDS}

Obstructive sleep apnea syndrome, genetic diseases, rare diseases, upper airway deformity, continuous positive airway pressure (CPAP)

\section{INTRODUCTION}

Classical childhood obstructive sleep apnea syndrome (OSAS) is a relatively frequent pathology, affecting $1 \%$ to $2 \%$ of children between the ages of 3 and 6 years ${ }^{10}$. Adenotonsillar hypertrophy is the primary cause. It is associated with nocturnal and diurnal signs, of variable severity. Nocturnal signs may include snoring, apneas, night-sweats, nycturia, parasomnia, agitated sleep and sometimes abnormal sleeping posture with the head in hyperextension. Diurnal signs comprise difficulty in awakening, irritability, hyperactivity, attention and memory disorder, asthenia, daytime somnolence, headache or vomiting, breakfast anorexia, oral breathing or growth disorder (only seen 
in the most severe forms). Neurocognitive dysfunction, which may be irreversible, is the most frequent and severe consequence of OSAS, hence the importance of early and adapted treatment $^{10}$. Cardiovascular (arterial hypertension) and metabolic consequences (metabolic syndrome)are less frequent and less severe in children than in adults. Classical OSAS is usually of moderate severity. Adenotonsillectomy is the first-line treatment, and is curative in the vast majority of cases, residual OSAS being mainly found in case of obesity or allergy?

OSAS is also found in a certain number of genetic diseases and/or malformations. The clinical presentation may in this case be more discreet or, in contrast, stronger, requiring special treatment. It is, however, important to be aware of the pathologies potentially underlying syndromic OSAS and thus to be in a position to prescribe systematic sleep exploration ahead of referral to a multidisciplinary specialist consultation.

\section{PATHOLOGIES POTENTIALLY UNDERLYING SYNDROMIC OSAS (Tab. 1)}

A large-scale epidemiological study performed in the State of Washington between 1987 and 2003 showed OSAS to be significantly more frequent in case of craniofacial abnormality (relative risk $[R R], 38$ ), facial cleft (RR, 40), Down's syndrome (RR, 51), or any other deformity $(R R, 4.1)^{8}$. Several other studies likewise reported increased prevalence of OSAS in case of craniofacial stenosis, achondroplasia or pycnodysostosis due to growth

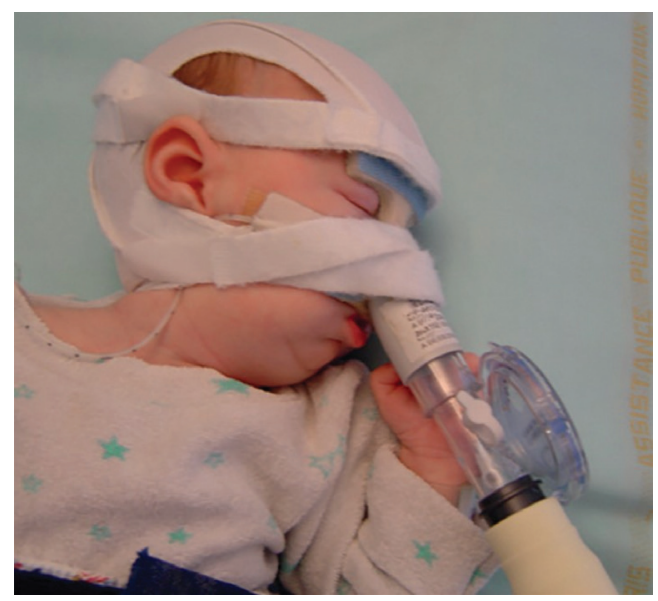

Figure 1

Infant with Pierre Robin

sequence, ventilated via a custom-made molded nasal mask.

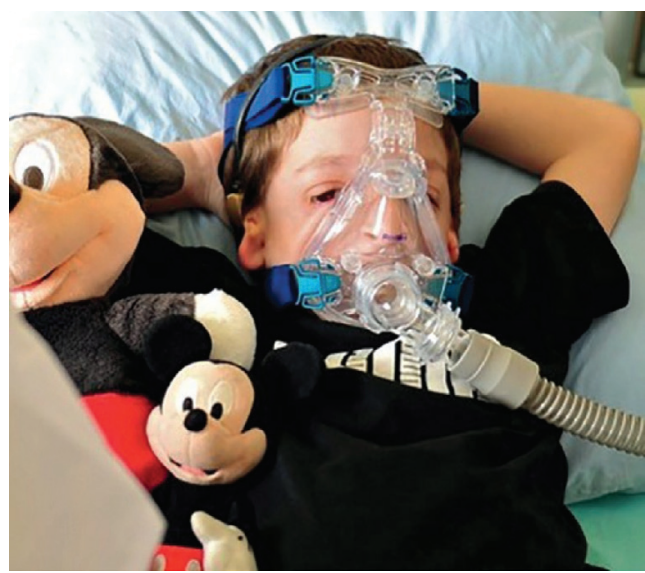

Figure 2

Boy with Treacher Collins syndrome, ventilated via an oronasal mask due to hypomandibulism and mouth breathing during sleep.

defect in the medial facial bones, causing anatomic narrowing of the upper airways. In a prospective study, our own team found that only 2 children out of 30 with achondroplasia showed normal polygraphy results ${ }^{6}$. Systematic sleep exploration in children and adults with Treacher Collins syndrome found that 4 children out of 8 had apnea-hypopnea indices (AHI) between 5 and 10/h and 1 had an $\mathrm{AHI}>10 / \mathrm{h}^{1}$. 
Table I: Comparison between classical and syndromic OSAS.

\begin{tabular}{|c|c|c|}
\hline & Classical OSAS & Syndromic OSAS \\
\hline Prevalence & $1-4 \%$ & all ages \\
\hline Age & $3-5$ years & >anatomic and dynamic obstacles \\
\hline Pathophysiology & adenotonsillar hypertrophy & severe \\
\hline Severity & moderate & absence of improvement \\
\hline Progression & variable & systematic PSG \\
\hline Risk factors & obesity-allergy-asthma & few studies \\
\hline Diagnosis & clinical \pm PSG & metabolic syndrome \\
\hline Consequences & $\begin{array}{c}\text { neurocognitive dysfunction cardiovascular stress } \\
\text { adenotonsillectomy }\end{array}$ & $\begin{array}{c}\text { specialist multidisciplinary team (surgery, ortho- } \\
\text { dontics, continuous positive pressure) }\end{array}$ \\
\hline Treatment & m
\end{tabular}

(OSAS: obstructive sleep apnea syndrome; PSG: polysomnography).

Sleep-disordered breathing is also very frequent in case of cleft palate, with severity increasing from isolated cleft to syndromic cleft to PierreRobinsequence $^{9}$ (Fig.1 and Fig. 2). A casecontrol study in children over 3 years of age with hemifacial microsomia treated in 26 American and Canadian craniofacial centers between 1993 and 2002 found significantly higher rates of snoring and sleep-disordered breathing in children with hemifacial microsomia than in controls ${ }^{4}$. OSAS is also an element in the clinical presentation associated with Prader-Willior

\section{DIAGNOSIS OF SYNDROMIC OSAS}

OSAS is more difficult to diagnose in syndromic children, as it may occur at any age, with greater frequency during periods of rapid facial growth such as infancy or adolescence. Moreover, without systematic sleep exploration, it may long go unidentified, as symptoms are chronic, mixed forms associating obstructive and central mechanisms are frequent, and the parents'
Rohhadnet syndrome, for which polysomnography should be systematic ${ }^{11}$. Finally, OSAS is also very frequent in Down's syndrome, due the associated flattened facial morphology, narrow nasal cavities and macroglossia, aggravated by tonsillar hypertrophy induced by relative immune deficiency ${ }^{3}$. In conclusion, syndromic childhood OSAS is less frequent than classical forms, but the number of potentially implicated syndromes is large, so that syndromic patients constitute a nonnegligible proportion of children with OSAS as a whole. perceptions are subjective. Several studies have further highlighted the frequent absence of clinical symptoms despite highly abnormal PSG findings, and a lack of correlation between clinical and anatomic or radiological signs. In achondroplasia, we reported a very weak correlation between clinical signs and PSG results ${ }^{6}$. One particularly interesting study compared sleep data in 12 infants with 
achondroplasia versus 12 controls, all aged less than 3 months ${ }^{5}$. The former had very disturbed sleep, with a high index for respiratory events compared to the controls. Paradoxically, however, their sleep showed normal efficacy, due to an abnormal reduction in arousal. These findings highlight the need for systematic sleep exploration in all at-risk children.

PSG is the reference examination for the diagnosis of respiratory disorders in children, although some authors perform only respiratory polygraphy in children with purely OSAS symptomatology. In syndromic OSAS, PSG is the reference examination, as obstructive and central mechanisms are frequently associated, with architectural abnormalities and impaired sleep quality. Central apnea is frequent in achondroplasia, cranial stenosis and mucopolysaccharidosis caused by compression of the cervicooccipital junction, and also in PraderWilli and Rohhadnet syndromes ${ }^{11}$.

\section{SEVERITY AND MORBIDITY IN SYNDROMIC OSAS}

Severity is often greater in syndromic than classical OSAS: obstruction is often multifactorial, combining anatomic (narrow airway) and dynamic elements (abnormal upper airway hypotonia), and frequently multi-level (nasal cavities, pharynx, larynx, trachea). For this reason, syndromic patients constitute a majority of those requiring long term continuous positive airway pressure (CPAP) treatment.

Associated morbidity has been less fully and more recently described in syndromic than in classical OSAS ${ }^{10}$. One of the difficulties is that these patients frequently present pathologies involving retarded psychomotor or intellectual development (Down's, Prader-Willi or Rohhadnet syndrome, mucopolysaccharidosis) in which intellectual and cognitive ability may be impaired due to associated sensory deficits (hearing loss, visual disorder). This highlights the absolute need for systematic exploration, to avoid falsely attributing an intellectual or neurocognitive deficit to the principal pathology whereas it could in fact be alleviated by preventing or correcting an OSAS.

\section{MANAGEMENT OF SYNDROMIC OSAS}

Management also differs between syndromic and classical OSAS. In the latter, adenotonsillectomy resolves OSAS in more than $80 \%$ of cases, whereas in syndromic OSAS head and neck surgery is often insufficient, contraindicated or impossible. Many of these patients are very young, and their OSAS is not related to any lymph tissue hypertrophy. Management thus needs to be multidisciplinary, associating a pediatric head and neck surgeon, pediatric maxillofacial surgeon, orthodontist, pediatric 
neurosurgeon and pediatric CPAP specialist. Treatment strategy is to be discussed on a case-by-case basis, according to the underlying pathology, patient's age and surgical possibilities. The fundamental point is that treatment options should be discussed in a specialized multidisciplinary team. Surgical interventions such as nasal calibration, turbinectomy mandibular distraction osteogenesis, or neurosurgical intervention may, for example, be an option in infants with craniofacial stenosis inducing severe OSAS. Sometimes, however, surgery is not feasible or is contraindicated due to the patient's age. It is in such cases that CPAP is indicated.

Non-invasive CPAP is a respiratory assistance technique that respects the airway, in contrast to tracheal intubation or tracheotomy. It consists in the delivery of a continuous positive airway pressure throughout the whole respiratory cycle, via a nasal or oronasal mask or nostril plugs. CPAP devices use a single circuit, with $\mathrm{CO}_{2}$ evacuated during expiration via a calibrated leak either in the interface or in the ventilator circuit. Many small, light, easy-to-use models are available for home use. They lack batteries and have limited alarm systems, but this is not mandatory as these patients do not need life support respiratory assistance. The air delivered is humidified and warmed in the nasal cavities, but in very young children a heating humidifier can improve tolerance and comfort, especially during prolonged use at night and for naps. The choice of interface depends on the patient's age, facial morphology and comfort and tolerance ${ }^{12}$, and is the main technical limitation in children with syndromic OSAS, as maxillofacial deformity is very frequent ${ }^{12}$. There are no validated criteria for initiating CPAP in syndromic OSAS; indications are generally obstructive sleep apnea and/or hypopnea on PSG, nocturnal gasometric abnormalities, with hypoxemia and hypercapnia, and clinical signs of severe obstruction of the upper airway. In young infants, insufficient weight gain may be the main symptom.

The aim of CPAP is to maintain sufficient airway opening throughout the respiratory cycle to normalize gas exchange and sleep quality. Pressure should therefore be targeted to the severity of laryngeal obstruction. We recently demonstrated that clinical criteria of resolution of stridor and signs of respiratory distress and normalization of nocturnal gas exchange underestimated the optimal CPAP level (i.e., the level best able to normalize or improve increased respiratory work) by about $2 \mathrm{~cm} \mathrm{H}_{2} \mathrm{O}^{7}$. In practice, it is therefore important to set pressure as high as tolerated by the child. For children, CPAP should be performed in a specialized multidisciplinary center with specific expertise. Education of parents and child takes only a few days, even for the youngest children. CPAP is initiated during daytime, preferably during a nap, then extended to nocturnal sleep. Adaptation generally takes 2-15 days, with a mean of 3-5 days depending on the child's age and compliance. A minimum 6 hours' continuous application should precede discharge home. Sleep recordings are made during the initial adaptation period; complete normalization of gasometric parameters and sleep quality is a 
requisite for discharge home. At home, surveillance is ensured by the home service provider, the nurse and/ or technician having pediatric training.
This highly effective treatment has considerably reduced resort to tracheotomy, which is now reserved to CPAP failure.

\section{CONCLUSION}

Childhood syndromic OSAS is a frequent pathology due to the numerous childhood conditions that may be associated. This highlights the need to know these risk-related pathologies to allow early screening and implement an adapted multidisciplinary management of sleep-disordered breathing.

Conflicts of interest: The authors declare no conflicts of interest.

\section{REFERENCES}

1. Akre H, Øverland B, Åsten P, Skogedal N, Heimdal K. Obstructive sleep apnea in Treacher Collins syndrome. Eur Arch Otorhinolaryngol 2012;269:331-7.

2. Bhattacharjee $R$, et al. Adenotonsillectomy outcomes in treatment of OSA in children: A multicenter retrospective study. Am J Respir Crit Care Med 2010;82:676-83.

3. Breslin J, Spanò G, Bootzin R, Anand P, Nadel L, Edgin J. Obstructive sleep apnea syndrome and cognition in Down syndrome. Dev Med Child Neurol 2014;56:657-64.

4. Cloonan YK, Kifle Y, Davis S, Speltz ML, Werler MM, Sta rr JR. Sleep outcomes in children with hemifacial microsomia and controls: a follow-up study. Pediatrics 2009;124:e313-e321.

5. Ednick M, Tinkle BT, Phro mchairak J, Egelhoff J, Amin R, Simakajornboon N. Sleeprelated respiratory abnormalities and arousal pattern in achondroplasia during early infancy. J Pediatr 2009;155:510-55.

6. Julliand $S$, et al. Lung function, diagnosis and treatment of sleep-disordered breathing and in children with achondroplasia. Am J Clin Genetics 2012;158A(8):1987-93.

7. Khirani S, Ramirez A, Aloui S, Leboulanger N, Picard A, Fauroux B. Continuous positive air pressure titration in infants with severe airway obstruction or bronchopu Imonary dysplasia. Crit Care 2013;17:R167.

8. Lam DJ, Jensen CC, Mueller BA, Starr JR, Cunningham ML, Weaver EM. Pediatric sleep apnea and craniofacial anomalies: a population-based casecontrol study. Lar yngoscope 2010;120:2098-105.

9. MacLean JE, Fitzsimons D, Fitzgerald DA, Waters KA. The spectrum of sleepdisordered breathing symptoms and respiratory events in infants with cleft lip and/or palate. Arch Dis Child 2012;97:1058-63.

10. Marcus $C L$, et al. Diagnosis and management of childhood obstructive sleep apnea syndrome. Pediatrics 2012;130:e714-755.

11. Miller J, Wagner M. Prader-Willi syndrome and sleepdisordered breathing. Pediatr Ann 2013;42:200-4.

12. Ramirez A, et al. Interfaces for long term noninvasive positive pressure ventilation in children. Intensi ve Care Med 2012;38:655-62. 